\title{
Peroxidase aus Meerrettich: \\ Kinetische Studien und Optimierung der Peroxidase-Aktivitätsbestimmung mit den Substraten $\mathrm{H}_{2} \mathrm{O}_{2}$ und 3,3',5,5'-Tetramethylbenzidin
}

\author{
Von H. Gallati und I. Pracht \\ Zentrale Forschungseinheiten F. Hoffmann-La Roche \& Co. AG Basel (Schweiz)
}

(Eingegangen am 14. Januar/29. April 1985)

\begin{abstract}
Zusammenfassung: Peroxidase aus Meerrettich wird oft als Markierungs- und Indikatorenzym bei Enzymimmunoassays eingesetzt. Zur Optimierung der Peroxidase-Aktivitätsbestimmung wird die Kinetik der katalytischen Reaktion in Abhängigkeit der $\mathrm{H}_{2} \mathrm{O}_{2}$ - und der 3,3',5,5'-Tetramethylbenzidin-Konzentration bei verschiedenen pH-Werten, Reaktionstemperaturen und Inkubationszeiten untersucht. Auf Grund dieser Ergebnisse wird ein Testverfahren vorgeschlagen, das erlaubt, die Peroxidase in einem Meßbereich von 0-200 ng/l mit einer Nachweisgrenze von $10 \mathrm{ng} / \mathrm{l}$ quantitativ zu bestimmen. Zusätzlich werden Meßmethoden zur Bestimmung von $3,3^{\prime}, 5,5^{\prime}$-Tetramethylbenzidin und $\mathrm{H}_{2} \mathrm{O}_{2}$ beschrieben.
\end{abstract}

Horseradish peroxidase:

Kinetic studies and optimization of the peroxidase activity determination with the substrates $\mathrm{H}_{2} \mathrm{O}_{2}$ and $3,3^{\prime}, 5,5^{\prime}$ tetramethylbenzidine

Summary: Horseradish peroxidase is often used as a labelling and indicator enzyme in enzyme immunoassays. In order to optimize the peroxidase activity determination, the kinetics of the catalytic reaction were investigated in relation to the concentration of $\mathrm{H}_{2} \mathrm{O}_{2}$ and 3,3',5,5'-tetramethylbenzidine, at different $\mathrm{pH}$ values, reaction temperatures and incubation times. On the basis of the results, a test procedure is presented, which enables the quantitative determination of peroxidase in the range $0-200 \mathrm{ng} / \mathrm{l}$. In addition, methods are described for the determination of 3,3',5,5'-tetramethylbenzidine and $\mathrm{H}_{2} \mathrm{O}_{2}$.

\section{Einfuihrung}

Peroxidase aus Meerrettich (Donor: hydrogen peroxide oxidoreductase, EC 1.11.1.7) wird häufig bei Enzymimmunoasssays als Markierungs- und Indikatorenzym verwendet.

Zur Bestimmung der Peroxidase steht mit o-Phenylendiamin ein hochempfindlicher Redoxindikator zur Verfügung. Allerdings besitzt dièses Substrat eine ausgeprägte mutagene und kanzerogene Aktivität.

Mit 3,3',5,5'-Tetramethylbenzidin wurde ein Redoxindikator synthetisiert (1), der keine oder nur sehr geringe mutagene und kanzerogene Aktivität zeigt (1,
2). Es konnte nachgewiesen werden, daß die Kanzerogenität des Benzidins erst durch die Hydroxylierung in ortho-Stellung des Moleküls entsteht. Durch die Methylierung des Benzidins ist die Bildung des 3,3'Dihydroxybenzidins im Organismus nicht mehr möglich, so daß es seine mutagene und kanzerogene Aktivität verliert.

$3,3^{\prime}, 5,5^{\prime}$-Tetramethylbenzidin wurde bis jetzt vor allem für die Bluttests, den Hämoglobinnachweis im Serum und im Urin verwendet, um das stark kanzerogene Benzidin zu ersetzen (3, 4). 3,3',5,5'-Tetramethylbenzidin eignet sich aber auch für die Bestimmung der Peroxidase (5). 
In der vorliegenden Arbeit wird zur Optimierung der Peroxidase-Äktivitätsbestimmung die Kinetik der katalytischen Reaktion in Abhängigkeit der $\mathrm{H}_{2} \mathrm{O}_{2}$ und der 3,3',5,5'-Tetramethylbenzidin-Konzentration bei unterschiedlichen $\mathrm{pH}$-Werten, Reaktionstemperaturen und Inkubationszeiten untersucht. Als Ergebnis kann ein einfaches Testverfahren vorgeschlagen werden, das erlaubt, die Peroxidase bei einem Meßbereich von $0-200 \mathrm{ng} / \mathrm{l}$ mit einer Nachweisgrenze von $10 \mathrm{ng} / \mathrm{l}$ quantitativ zu bestimmen.

Zusätzlich wurde die Möglichkeit zum sofortigen und vollständigen Abstoppen der peroxidatischen Aktivität sowie zur Stabilisierung der entstandenen Farbintensität untersucht. Es werden auch Testmethoden zur Bestimmung der 3,3',5,5'-Tetramethylbenzidinund der $\mathrm{H}_{2} \mathrm{O}_{2}$-Konzentration beschrieben.

\section{Material und Methoden}

\section{Reagenzien}

Peroxidase aus Meerrettich (Donor: hydrogen-peroxide oxidoreductase, EC 1.11.1.7) wurde in lyophilisierter Form von der Firma Boehringer Mannheim (Best. Nr. 108090) bezogen. Das Enzympräparat, dessen Reinheitszahl mit „ca 3,0" (Extinktion $403 \mathrm{~nm} /$ Extinktion $275 \mathrm{~nm}$ ) angegeben wird, wurde ohne weitere Reinigung eingesetzt.

3,3'.5,5'-Tetramethylbenzidin wurde von der Firma Fluka Schweiz (Best. Nr. 87748) und $\mathrm{H}_{2} \mathrm{O}_{2}$ in einer $300 \mathrm{~g} / \mathrm{kg}$-Lösung von der Firma Merck (Best. Nr. 822287) geliefert.

Die übrigen Chemikalien waren von analytischer Reinheit.

\section{Testlösungen}

$\mathrm{H}_{2} \mathrm{O}_{2}$-Pufferlösung: Die $\mathrm{H}_{2} \mathrm{O}_{2}$-Pufferlösung enthält $0,21 \mathrm{~mol} / \mathrm{l}$ Kaliumcitrat vom pH 3,95 $( \pm 0,05)$ und $3,15 \mathrm{mmol} / 1 \mathrm{H}_{2} \mathrm{O}_{2}$ Diese Lösung ist, bei $15-25^{\circ} \mathrm{C}$ aufbewahrt, einige Wochen haltbar.

3,3',5,5'-Tetramethylbenzidin: $252 \mathrm{mg} 3,3^{\prime}, 5,5^{\prime}$-Tetramethylbenzidin $\left(M_{\mathrm{r}}=240,35\right)$ werden in $5,0 \mathrm{ml}$ Aceton gelöst und anschließend $45 \mathrm{ml}$ Methanol zugegeben. Diese $21 \mathrm{mmol} / 1$ 3, 3',5,5'Tetramethylbenzidin-Lösung ist lichtgeschützt bei $15-25^{\circ} \mathrm{C}$ aufbewahrt einige Wochen haltbar.

\section{Aktivitätsbestimmung der Peroxidase}

Zur Herstellung der gebrauchsfertigen Substratlösung wird zu 20 Volumenteilen $\mathrm{H}_{2} \mathrm{O}_{2}$-Pufferlösung 1 Volumenteil 3,3',5,5'Tetramethylbenzidin-Lösung zugemischt. Diese Lösung ist bei $15-25^{\circ} \mathrm{C}$ etwa $2-3$ Stunden haltbar.

Für die Aktivitätsbestimmung der Peroxidase werden in einer Photometer-Meßküvette von $10 \mathrm{~mm}$ Schichtdicke zu $1,0 \mathrm{ml}$ gebrauchsfertiger Substratlösung $(0,2 \mathrm{~mol} / 1 \mathrm{Kaliumcitrat}$ vom pH 3,95 mit $3 \mathrm{mmol} / 1 \mathrm{H}_{2} \mathrm{O}_{2}$ und $1 \mathrm{mmol} / 1$ 3,3',5,5'-Tetramethylbenzidin) $0,1 \mathrm{ml}$ Peroxidaselösung (Endkonzentration in der Testlösung bis $200 \mu \mathrm{g} / 1$ Peroxidase) zugemischt und bei $22^{\circ} \mathrm{C}$ die Absorptionszunahme während 30 Minuten bei der Wellenlänge $370 \mathrm{~nm}$ photometrisch gemessen. Abweichungen von diesem Testvorgehen werden bei den einzelnen Versuchen speziell erwähnt.

\section{Resultate und Diskussion}

Struktur, Reaktionsmechanismus und Spektren des $3,3^{\prime}, 5,5^{\prime}$-Tetramethylbenzidins

In Anlehnung an die Arbeit von P. D. Josephy et al. (6) über die Oxidation des $3,3^{\prime}, 5,5^{\prime}$-Tetramethylbenzidins kann angenommen werden, daß áuch unter der katalytischen Einwirkung der Peroxidase das farblose $3,3^{\prime}, 5,5^{\prime}$-Tetramethylbenzidin (I) in einem ersten Schritt zum blaugefärbten Radikalkation (II) und dieses in einem zweiten Schritt zum gelbgefärbten 3,3',5,5'-Tetramethyl-1,1' -diphenochinon-4-4'-diimoniumsalz (III) oxidiert wird.

I. 3,3',5,5'-Tetramethylbenzidin, farblos, $\lambda_{\max }=$ $210 \mathrm{~nm}, 286 \mathrm{~nm}$<smiles>Cc1cc(-c2cc(C)c(N)c(C)c2)cc(C)c1N</smiles>

II. $\quad 3,3^{\prime}, 5,5^{\prime}$-Tetramethylbenzidin-Radikalkation, blaugefärbt, $\lambda_{\max }=210 \mathrm{~nm}, 370 \mathrm{~nm}, 650 \mathrm{~nm}$

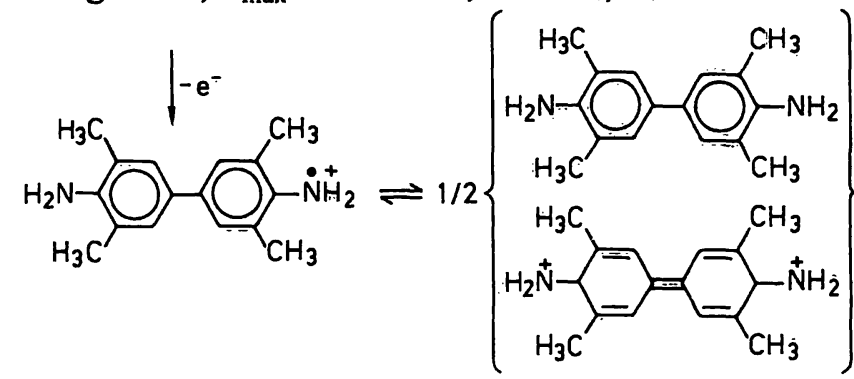

III. 3,3',5,5'-Tetramethyl-1,1'-diphenochinon-4,4'-diimoniumion, gelbgefärbt, $\lambda_{\text {max }}=210 \mathrm{~nm}, 450 \mathrm{~nm}$<smiles></smiles>

In Abbildung 1 ist das Spektrum des 3,3',5,5'-Tetramethylbenzidins (I) mit den zwei ausgeprägten Absorptionsmaxima bei den Wellenlängen $210 \mathrm{~nm}$ und $286 \mathrm{~nm}$ aufgezeichnet. Während dem ersten Oxidationsschritt verschwindet die Absorbanz bei der Wellenlänge $286 \mathrm{~nm}$, während jene bei $210 \mathrm{~nm}$ erhalten bleibt. Die Konzentration an 3,3',5,5'-Tetramethylbenzidin (I) kann demnach mit einer einfachen $A b-$ sorptionsmessung bei der Wellenlänge $286 \mathrm{~nm}$ bestimmt werden: In einer Quarzküvette von $10 \mathrm{~mm}$ Schichtdicke wird vom $3,3^{\prime}, 5,5^{\prime}$-Tetramethylbenżidin $(0-50 \mu \mathrm{mol} / \mathrm{l})$, das in bidestilliertem.Wasser gelöst ist, die Absorbanz bei der Wellenlänge $286 \mathrm{~nm}$ gemessen. An Hand einer mitgeführten Standardkurve kần direkt die 3,3',5,5'-Tetramethylbenzidin-Konzentration in der Probe abgelesen werden. 


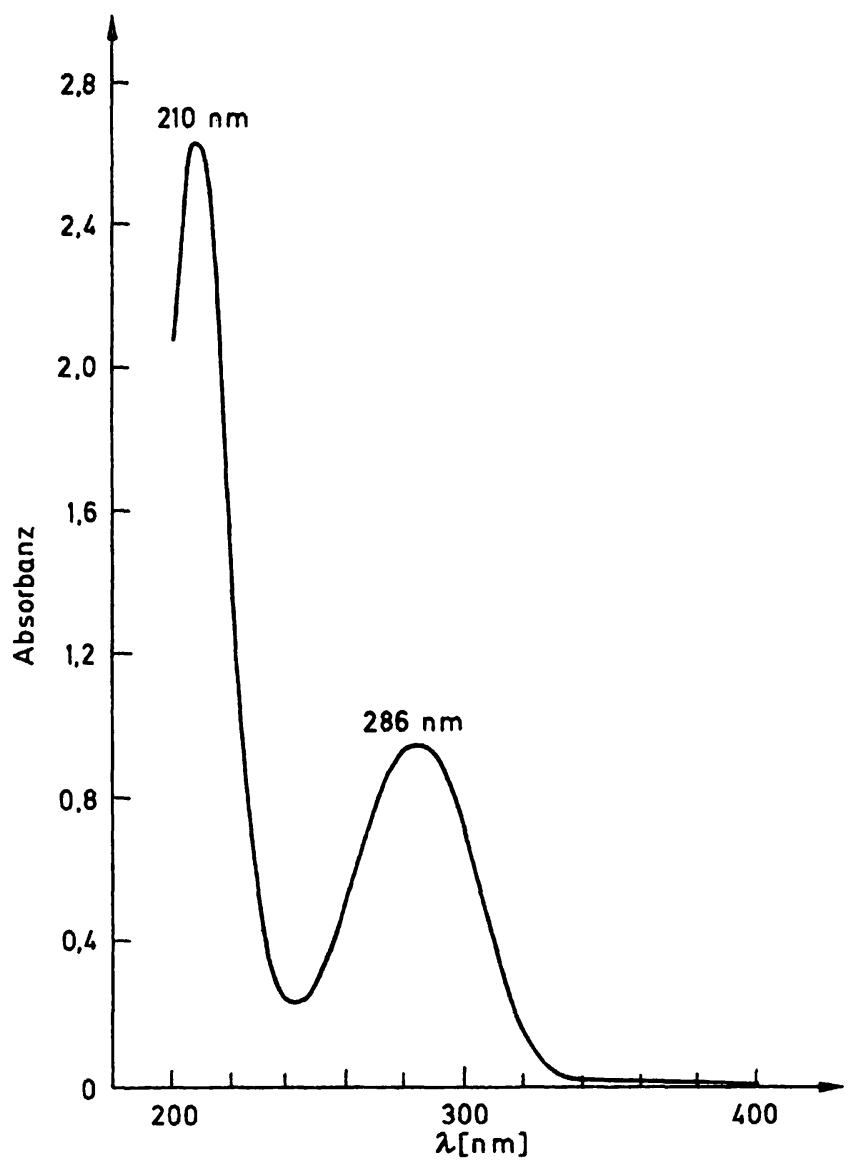

Abb. 1. Absorptionsspektrum des 3,3',5,5'-Tetramethylbenzidin (I).

Von $30 \mu \mathrm{mol} / 13,3^{\prime}, 5,5^{\prime}$-Tetramethylbenzidin in destilliertem Wasser wurde das Spektrum im Wellenlängenbereich von $200 \mathrm{~nm}-400 \mathrm{~nm}$ aufgenommen.

Abbildung 2 zeigt die Absorptionsspektren, wie sie während der 30-minütigen enzymatischen Oxidation des $3,3^{\prime}, 5,5^{\prime}$-Tetramethylbenzidins jeweils nach bestimmten Zeitintervallen aufgenommen wurden. Unter den gegebenen Reaktionsbedingungen (geringe Peroxidasemenge, großer Überschuß an $3,3^{\prime}, 5,5^{\prime}-$ Tetramethylbenzidin und $\mathrm{H}_{2} \mathrm{O}_{2}$ ) wird nur das blaugefärbte Radikalkation (II) mit den Absorptionsmaxima bei den Wellenlängen $370 \mathrm{~nm}$ und $650 \mathrm{~nm}$ gebildet. Auf Grund dieser Resultate kann für die kinetische Aktivitätsbestimmung der Peroxidase die photometrische Messung sowohl bei $640-650-660 \mathrm{~nm}$ wie auch bei $370 \mathrm{~nm}$ durchgeführt werden. Die Absorbanz des Radikalkations' (II) bei der Wellenlänge $370 \mathrm{~nm}$ ist um $60 \%$ höher als bei der Wellenlänge $650 \mathrm{~nm}$.

Mit einer drastischen Erhöhung der Peroxidasemenge und/oder einer entsprechenden Verminderung der $3,3^{\prime}, 5,5^{\prime}$-Tetramethylbenzidin-Konzentration in der Testlösung kann neben dem blaugefärbten Radikalkation (II) mehr und mehr auch das gelbgefärbte $3,3^{\prime}, 5,5^{\prime}$-Tetramethyl-1,1'-diphenochinon-4,4'-diimo-

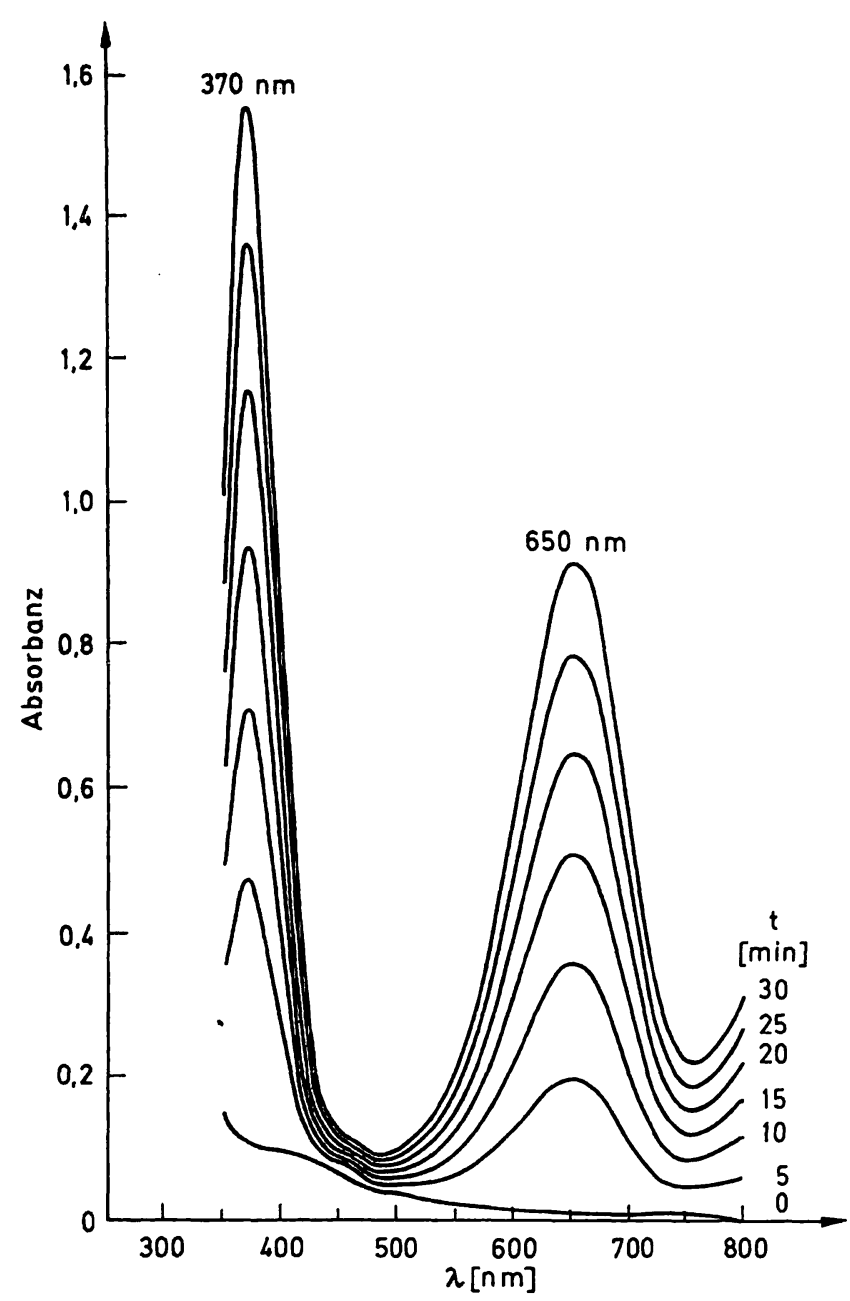

Abb. 2. Absorptionsspektrum des 3,3',5,5'-Tetramethylbenzidins während der enzymatischen Oxidation. $200 \mathrm{pg}$ Peroxidase werden in $1,0 \mathrm{ml}$ Substratpuffer $(0,2$ mol/l Kaliumcitrat von $\mathrm{pH} \mathrm{3,95} \mathrm{mit} 3 \mathrm{mmol} / 1 \mathrm{H}_{2} \mathrm{O}_{2}$ und $1,0 \mathrm{mmol} / 13,3^{\prime}, 5,5^{\prime}$-Tetramethylbenzidin) bei $22^{\circ} \mathrm{C}$ inkubiert und während der Reaktionsdauer von 30 Minuten jeweils nach je 5 Minuten ein Absorptionsspektrum im Wellenlängenbereich von $350 \mathrm{~nm}$ $-800 \mathrm{~nm}$ aufgenommen.

niumion (III) gebildet werden. In Abbildung 3 sind die Absorptionsspektren dargestellt, wie sie mit unterschiedlichen Peroxidasemengen in der Testlösung $(0,03-20 \mathrm{mg} / \mathrm{l})$ bei Anwesenheit von $15 \mu \mathrm{mol} / 13,3^{\prime}, 5,5^{\prime}-$ Tetramethylbenzidin erhalten wurden. Diese Resultate zeigen, da $\beta$ mit steigender Peroxidasekonzentration die Absorption bei den Wellenlängen $370 \mathrm{~nm}$ und $650 \mathrm{~nm}$ abnimmt, während sich gleichzeitig ein neues Absorptionsmaximum bei der Wellenlänge $450 \mathrm{~nm}$ bildet. Die Abbildung 4 zeigt an Hand der Absorptionsspektren die zeitabhängige, enzymatische Oxidation des 3,3',5,5'-Tetramethylbenzidins (I) über das Radikalkation (II) zum 3,3',5,5'-Tetramethyl-1,1'-diphenochinon-4,4'-diimoniumion (III) bei Anwesenheit von $15 \mu \mathrm{mol} / 13,3^{\prime}, 5,5^{\prime}$-Tetramethylbenzidin und $4 \mathrm{mg} / 1$ Peroxidase in der Testlösung. 


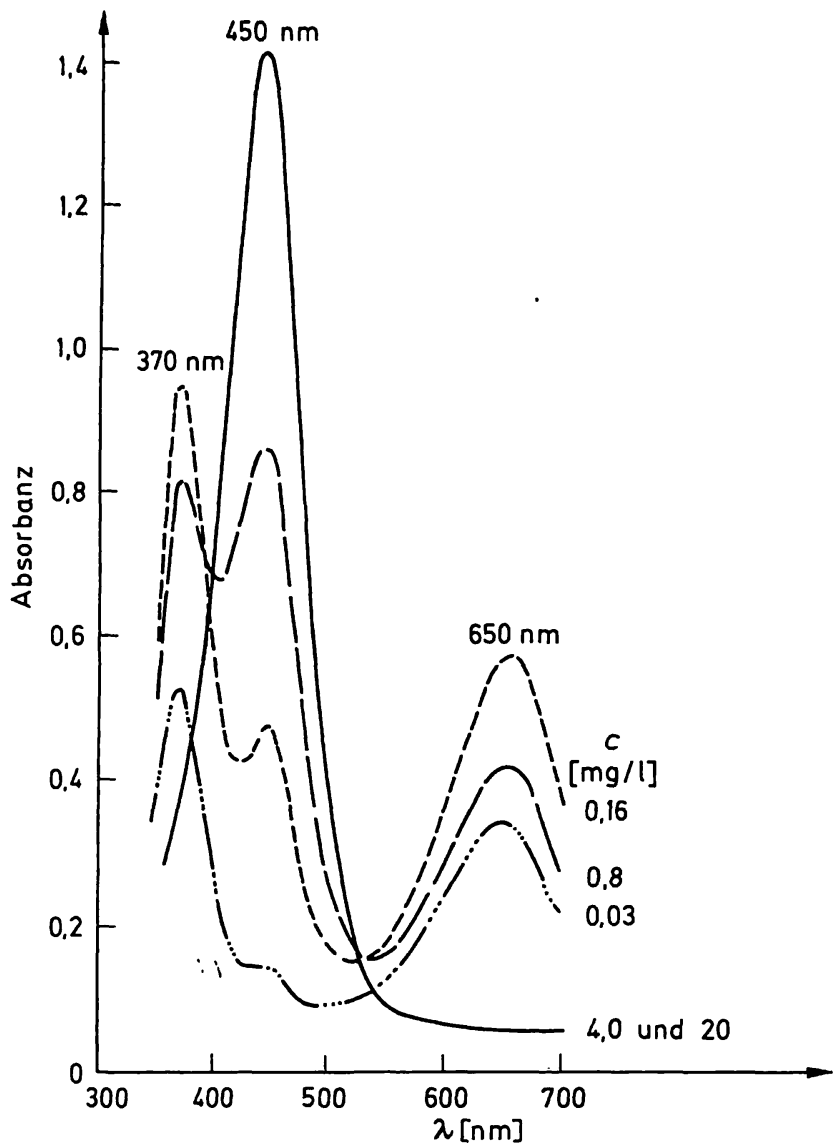

Abb. 3. Absorptionsspektren des 3,3',5,5'-Tetramethylbenzidins, das unter Einwirkung verschiedener Mengen Peroxidase oxidiert wurde.

$15 \mu \mathrm{mol} / 13,3^{\prime}, 5,5^{\prime}$-Tetramethylbenzidin in $0,2 \mathrm{~mol} / \mathrm{l} \mathrm{Ka-}$ liumcitratpuffer vom $\mathrm{pH} 3,95$ wurde mit $3 \mathrm{mmol} / 1 \mathrm{H}_{2} \mathrm{O}_{2}$ in Anwesenheit von unterschiedlichen Peroxidasekonzentrationen $(0,03-20 \mathrm{mg} / \mathrm{l})$ während einer 30 -minütigen Reaktionsdauer bei $22^{\circ} \mathrm{C}$ zur Umsetzung gebracht und anschließend die Absorptionsspektren der einzelnen Testlösungen im Wellenlängenbereich von $350 \mathrm{~nm}-700 \mathrm{~nm}$ aufgenommen.

Auf Grund dieser Verhältnisse kann die Konzentration an 3,3',5,5'-Tetramethylbenzidin (I) auch enzymatisch bestimmt werden: In einer Photometerküvette von $10 \mathrm{~mm}$ Schichtdicke werden $0-20 \mu \mathrm{mol} / \mathrm{l}$ $3,3^{\prime}, 5,5^{\prime}$-Tetramethylbenzidin in $0,2 \mathrm{~mol} / 1 \mathrm{Kaliumci-}$ tratpuffer von $\mathrm{pH} 3,95$ bei Anwesenheit von 3,0 $\mathrm{mmol} / 1 \mathrm{H}_{2} \mathrm{O}_{2}$ durch Zusatz von $20 \mathrm{mg} / 1$ Peroxidase katalytisch zum $3,3^{\prime}, 5,5^{\prime}$-Tetramethyl-1,1'-diphenochinon-4,4'-diimoniumion oxidiert und die Absorbanz bei der Wellenlänge $450 \mathrm{~nm}$ gemessen. An Hand einer mitgeführten Standardkurve kann direkt die $3,3^{\prime}, 5,5^{\prime}$-Tetramethylbenzidin-Konzentration in der Probe abgelesen werden.

Das oxidierte, blaugefärbte $3,3^{\prime}, 5,5^{\prime}$-Tetramethylbenzidin-Radikalkation (II) kann auch durch die Senkung des $\mathrm{pH}$-Wertes zum gelben 3,3',5,5' -Tetramethyl-1, $1^{\prime}$-diphenochinon-4, $4^{\prime}$-diimoniumion

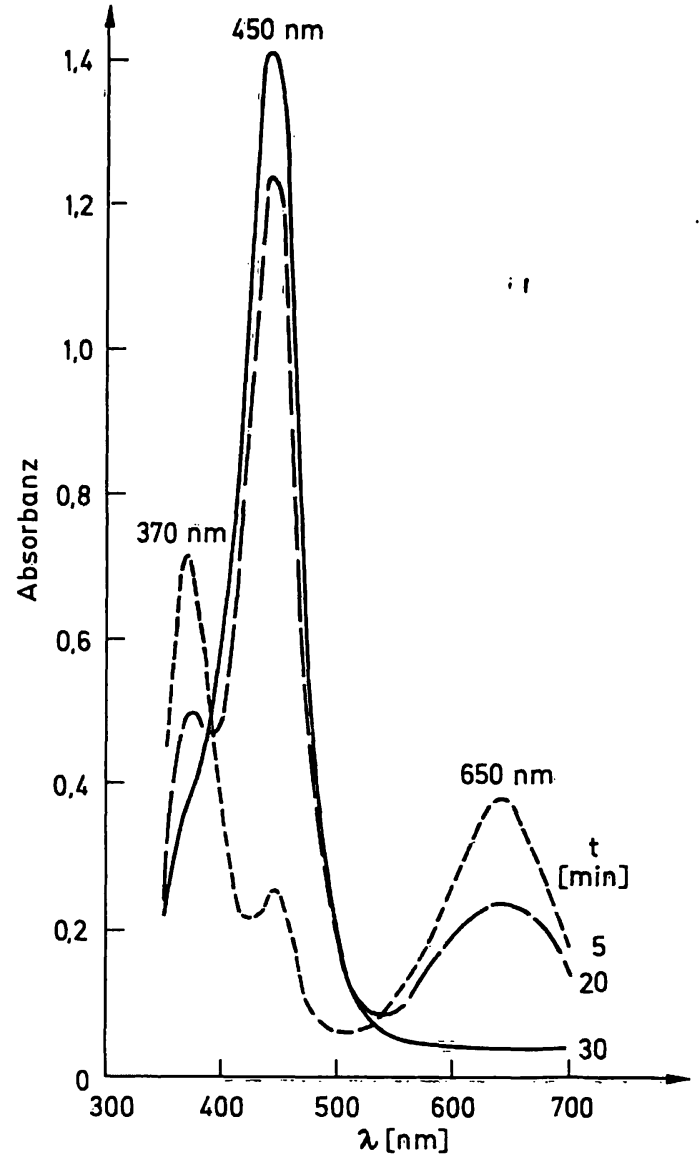

Abb. 4. Absorptionsspektren des 3,3',5,5'-Tetramethylbenzidins während der enzymatischen Oxidation bei Anwesenheit von $4 \mathrm{mg} / 1$ Peroxidase in der Testlösung. $15 \mu \mathrm{mol} / 13,3^{\prime}, 5,5^{\prime}$-Tetramethylbenzidin in $0,2 \mathrm{~mol} / 1 \mathrm{Ka}-$ liumcitratpuffer vom $\mathrm{pH} 3,95$ wurde mit $3,0 \mathrm{mmol} / \mathrm{l}$ $\mathrm{H}_{2} \mathrm{O}_{2}$ in Anwesenheit von $4,0 \mathrm{mg} / \mathrm{l}$ Peroxidase bei $22^{\circ} \mathrm{C}$ zur Umsetzung gebracht, wobei während der 30-minütigen Reaktionsdauer nach der 5., der 20. und der 30. Minute je ein Absorptionsspektrum im Wellenlängenbereich von $350 \mathrm{~nm}-700 \mathrm{~nm}$ aufgenommen wurde.

überführt werden. Wie die Absorptionsspektren der Abbildung 5 zeigen, ist dabei der Anteil an 3,3',5,5'Tetramethyl-1,1'-diphenochinon-4,4'-diimoniumion (III) zum Radikalkation (II) von der Wasserstoffionenkonzentration abhängig. Eine vollständige Umsetzung zum 3,3',5,5'-Tetramethyl-1,1'-diphenochinon-4,4'-diimoniumion und damit zusammenhängend die Ausbildung eines einzigen Absorptionsmaximums bei der Wellenlänge $450 \mathrm{~nm}$ wird bei einem $\mathrm{pH}=$ Wert von 1,0 und tiefer erreicht. Dies ist vor allem bei der Durchführung von enżyma-immunologischen Tests, bei denen die Peroxidase als Indikatorenzym eingesetzt wird, von großem Vorteil. Durch die Absenkung des $\mathrm{pH}$-Wertes in der Testlösung kann zugleich die peroxidatische Aktivität sofort und vollständig gestoppt, die Farbintensität gesteigert und stabilisiert sowie eine Farbe erreicht werden, für deren photometrische Messung das gebräuchliche Filter von $450 \mathrm{~nm}$ benützt werden kaṇin. 


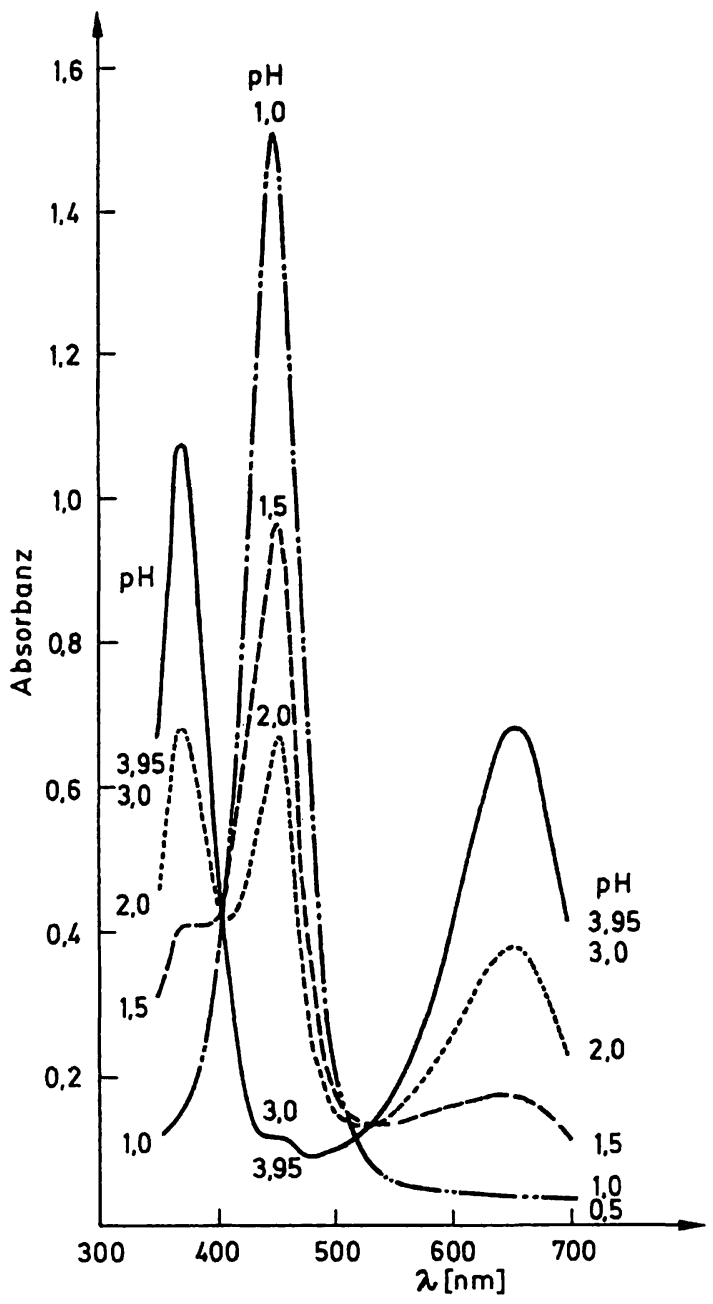

Abb. 5. Absorptionsspektren des oxidierten 3,3',5,5'-Tetramethylbenzidins bei verschiedenen pH-Werten.

Nach einer 30-minütigen katalytischen Einwirkung von $150 \mathrm{ng} / 1$ Peroxidase auf $1,0 \mathrm{mmol} / 13,3^{\prime}, 5,5^{\prime}$-Tetramethylbenzidin in Anwesenheit von $3 \mathrm{mmol} / 1 \mathrm{H}_{2} \mathrm{O}_{2}$ in $0,2 \mathrm{~mol} / \mathrm{l}$ Kaliumcitratpuffer vom pH 3,95 wurde durch Zugabe von konzentrierter Schwefelsäure der pH-Wert der Testlösung entsprechend abgesenkt auf $\mathrm{pH} 3,0$ $(-)$, auf $\mathrm{pH} 2,0(\cdots \cdots), \mathrm{pH} \mathrm{1,5}(---)$ und dann auf $\mathrm{pH} 1,0$ und $0,5(-. .-)$. Von diesen Lösungen sind die Absorptionsspektren im Wellenlängenbereich von $350 \mathrm{~nm}-700 \mathrm{~nm}$ aufgenommen.

Peroxidaseaktivität in Abhängigkeit der $3,3^{\prime}, 5,5^{\prime}$-Tetramethylbenzidin-

\section{Konzentration}

Die Abhängigkeit der Peroxidaseaktivität von der $3,3^{\prime}, 5,5^{\prime}$-Tetramethylbenzidin=Konzentration ist in der Abbildung 6 nach der Darstellungsweise von $L i$ neweaver-Burk aufgezeichnet. Daraus kann direkt die 3,3',5,5'-Tetramethylbenzidin-Affinität der Peroxidase als Michaelis-Konstante $\left(K_{\mathrm{m}}=0,31 \mathrm{mmol} / \mathrm{l}\right)$ sowie die maximal mögliche Reaktionsgeschwindigkeit abgelesen werden. Auf Grund der beschränkten Löslichkeit im Substratpuffer sollte allerdings diè $3,3^{\prime}, 5,5^{\prime}$-Tetramethylbenzidin-Konzentration von 1 $\mathrm{mmol} / \mathrm{l}$ nicht überschritten werden. Die 3,3',5,5'-Tetra-

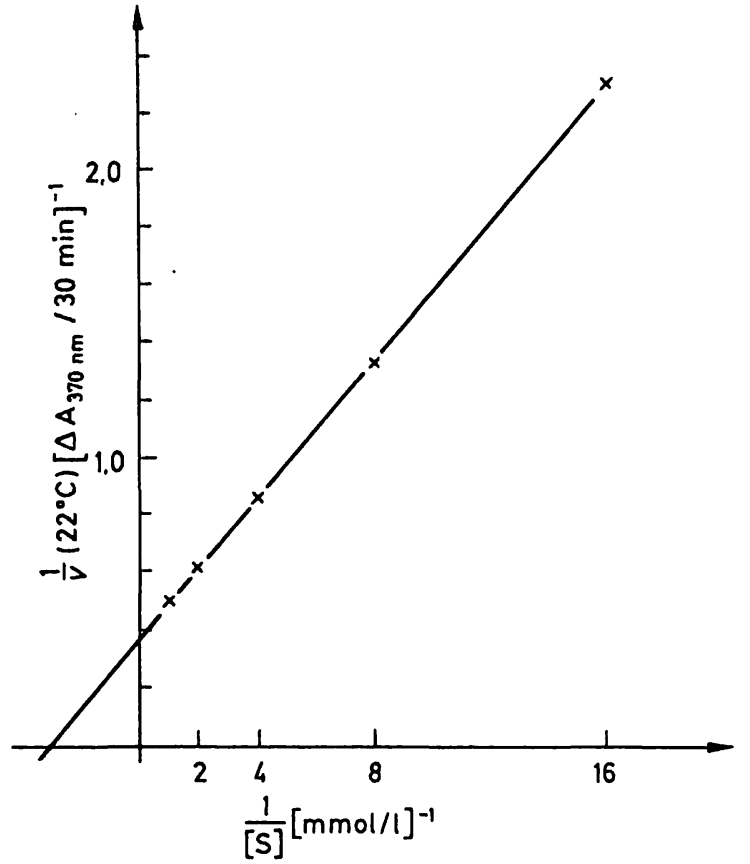

Abb. 6. Michaelis-Konstante der Peroxidase fürs 3,3',5,5'-Tetramethylbenzidin.

$200 \mathrm{ng} / \mathrm{l}$ Peroxidase wurde während 30 Minuten bei $22^{\circ} \mathrm{C}$ in $0,2 \mathrm{~mol} / 1$ Kaliumcitratpuffer vom $\mathrm{pH} 3,95 \mathrm{mit}$ $3 \mathrm{mmol} / 1 \mathrm{H}_{2} \mathrm{O}_{2}$ und den entsprechenden $3,3^{\prime}, 5,5^{\prime}$-Tetramethylbenzidin-Konzentrationen inkubiert und anschließend die Absorbanz bei der Wellenlänge $370 \mathrm{~nm}$ photometrisch gemessen. Darstellung der Resultate nach Lineweaver-Burk.

methylbenzidin-Affinität der Peroxidase ist im untersuchten $\mathrm{pH}$-Bereich von 3,5-6,0 unabhängig vom pH-Wert der Testlösung.

\section{Peroxidaseaktivität in Abhängigkeit der $\mathrm{H}_{2} \mathrm{O}_{2}$-Konzentration}

Die Abhängigkeit der Peroxidaseaktivität von der $\mathrm{H}_{2} \mathrm{O}_{2}$-Konzentration wird sehr stark beeinflußt vom pH-Wert der Testlösung, von der Reaktionsdauer der peroxidatischen Oxidation sowie von der 3,3',5,5'Tetramethylbenzidin-Konzentration.

In Abbildung 7 sind die pH-Aktivitätskurven der Peroxidase bei Anwesenheit unterschiedlicher $\mathrm{H}_{2} \mathrm{O}_{2-}$ Konzentrationen dargestellt. Mit steigendem $\mathrm{H}_{2} \mathrm{O}_{2}$ Gehalt in der Testlösung ist eine deutliche Verschiebung des jeweiligen $\mathrm{pH}-$ Optimums gegen den sauren $\mathrm{pH}$-Bereich festzustellen. Unter den gegebenen Reaktionsbedingungen wird die höchste Peroxidaseaktivität mit einer $\mathrm{H}_{2} \mathrm{O}_{2}$-Konzentration von $1-3 \mathrm{mmol} / \mathrm{l}$ bei einem pH-Wert im Bereich von 3,8-4,4 erreicht.

Werden die Resultate der Abbildung 7 nach der Darstellungsweise von Lineweaver-Burk aufgezeichnet (Abb. 8), so kann für die untersuchten pH-Werte sowohl die maximal mögliche Reaktionsgeschwindigkeit als auch die $\mathrm{H}_{2} \mathrm{O}_{2}$-Affinität der Peroxidase als Michaelis-Konstante $\left(K_{\mathrm{m}}\right)$ direkt abgelesen werden. 


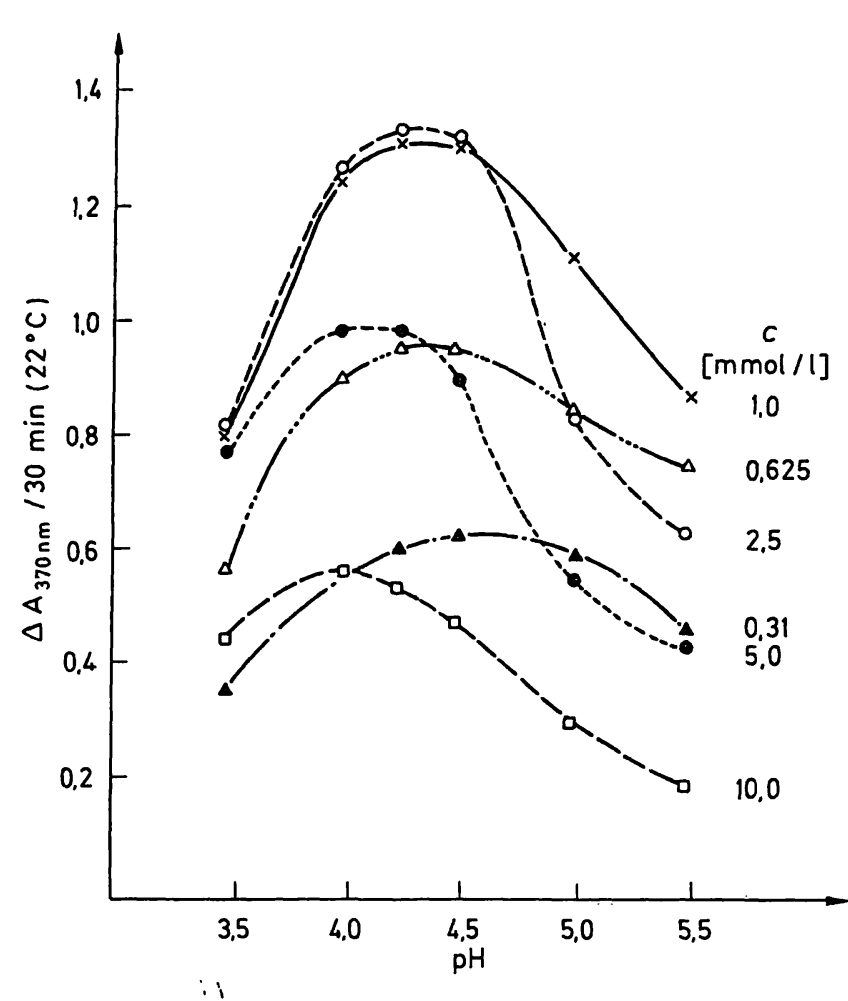

Abb. 7. pH-Aktivitätskurven der Peroxidase bei Anwesenheit verschiedener $\mathrm{H}_{2} \mathrm{O}_{2}$-Konzentrationen.

$200 \mathrm{ng} / \mathrm{l}$ Peroxidase wurde während 30 Minuten bei $22{ }^{\circ} \mathrm{C}$ in $0,2 \mathrm{~mol} / \mathrm{l} \mathrm{Kaliumcitratpuffer}$ bei den angegebenen $\mathrm{pH}$-Werten mit $1,0 \mathrm{mmol} / \mathrm{l}$ 3,3',5,5'-Tetramethylbenzidin und unterschiedlichen $\mathrm{H}_{2} \mathrm{O}_{2}$-Konzentrationen inkubiert und anschließend die Absorbanz bei der Wellenlänge $370 \mathrm{~nm}$ photometrisch gemessen.

Im $\mathrm{pH}$-Bereich von 3,5-4,5 nimmt die $\mathrm{H}_{2} \mathrm{O}_{2}$-Affinität der Peroxidase stark ab:

pH 3,5: $\quad K_{\mathrm{m}}=0,85 \mathrm{mmol} / 1$,

pH 4,0: $K_{\mathrm{m}}=1,58 \mathrm{mmol} / \mathrm{l}$,

pH 4,25: $K_{\mathrm{m}}=2,51 \mathrm{mmol} / \mathrm{l}$,

$\mathrm{pH} 4,5: \quad K_{\mathrm{m}}=3,33 \mathrm{mmol} / \mathrm{l}$.

Im $\mathrm{pH}$-Bereich von 4,5-6,0 ist die Affinität der Peroxidase zum $\mathrm{H}_{2} \mathrm{O}_{2}$ identisch und zeigt eine MichaelisKonstante $K_{\mathrm{m}}=3,33 \mathrm{mmol} / \mathrm{l}$.

In Abbildung 9 sind die Zeit-.Aktivitätskurven der Peroxidase bei Anwesenheit unterschiedlicher $\mathrm{H}_{2} \mathrm{O}_{2-}$ Konzentrationen in der Testlösung dargestellt. Diese Resultate zeigen, daß mit steigender $\mathrm{H}_{2} \mathrm{O}_{2}$-Konzentration einerseits eine höhere Anfangsaktivität der eingesetzten Peroxidase festzustellen ist, daß aber andererseits während der Reaktionsdauer die Peroxidaseaktivität schneller abnimmt, bedingt durch die $\mathrm{H}_{2} \mathrm{O}_{2}$-Inaktivierung des Enzyms. Zur Festsetzung der optimalen $\mathrm{H}_{2} \mathrm{O}_{2}$-Konżentration muß demnach auch die Inkubationsdauer der Peroxidase in der Testlösung berücksichtigt werden.

Das 3,3',5,5'-Tetramethylbenzidin-Peroxidase-System kann auch zur quantitativen Bestimmung von $\mathrm{H}_{2} \mathrm{O}_{2}$. eingesetzt werden: In einer Photometerküvette von

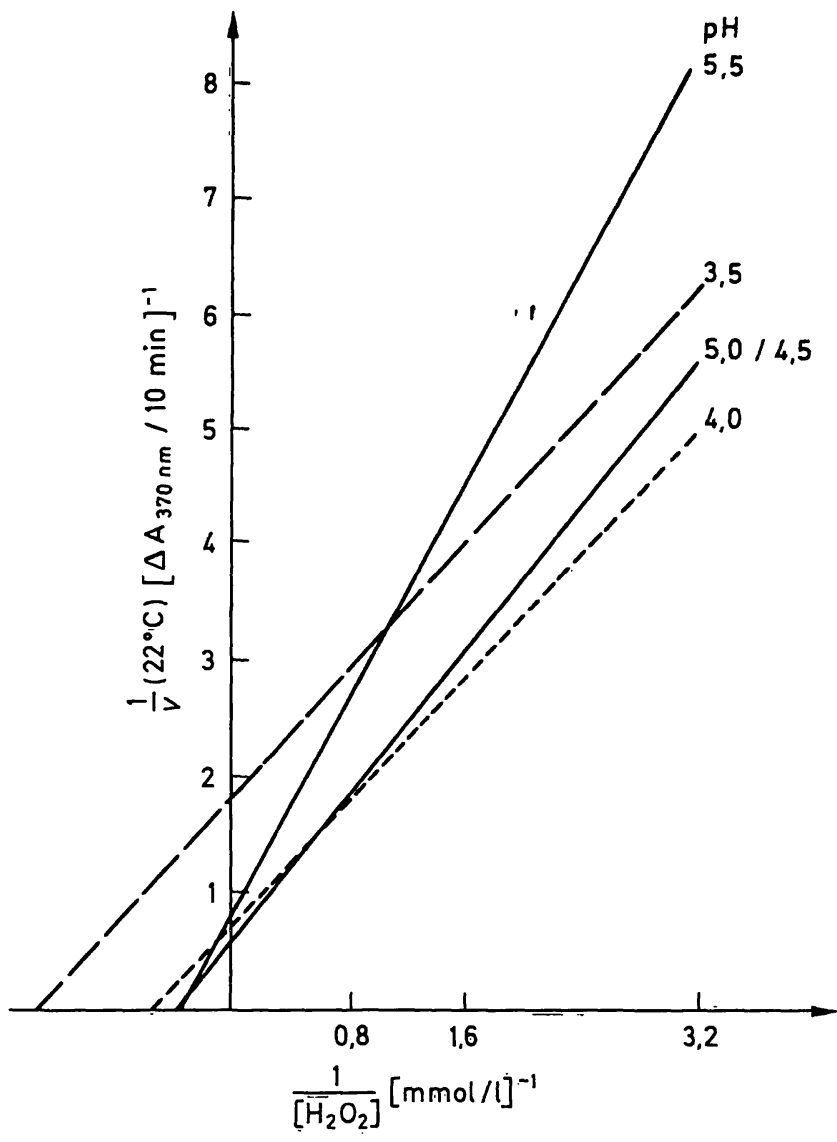

Abb. 8. Michaelis-Konstante der Peroxidase für $\mathrm{H}_{2} \mathrm{O}_{2}$ bei verschiedenen $\mathrm{pH}$-Werten.

Die Resultate der Abbildung 7 nach der Darstellungsweise von Lineweaver-Burk.

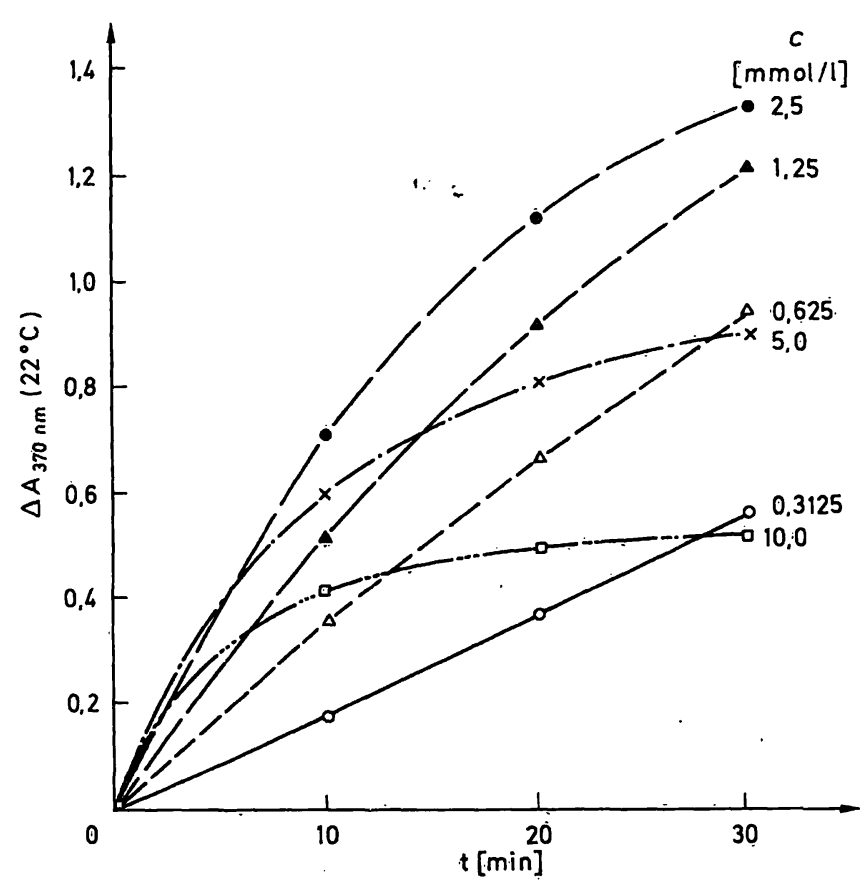

Abb. 9. Peroxidaseaktivität in Abhängigkeit zur $\mathrm{H}_{2} \mathrm{O}_{2}$-Konzentration und zuur Reaktionsdauer.

$200 \mathrm{ng} / \mathrm{l}$ Peroxidase würdèn bei einer Reaktionștemperatur von $22^{\circ} \mathrm{C}$ in $0,2 \mathrm{~mol} / \mathrm{l}$ Kaliumcitratpuffer vom pH 3,95 mit 1,0 mmol/1 3,3',5,5':Tetramethylbenzidin und verschiedenen $\mathrm{H}_{2} \mathrm{O}_{2}$-Konżentrationen inkubiert, wobei die Absorbanz der Testlösung bei der Wellenlänge $370 \mathrm{~nm}$ während dèr 30-minütigen Reaktion verfolgt wurde. 
$10 \mathrm{~mm}$ Schichtdicke werden $0-40 \mu \mathrm{mol} / 1 \mathrm{H}_{2} \mathrm{O}_{2}$ in 0,2 mol/1 Kaliumcitratpuffer vom $\mathrm{pH} 3,95$ bei Anwesenheit von $1,0 \mathrm{mmol} / 13,3^{\prime}, 5,5^{\prime}$-Tetramethylbenzidin durch Zugabe von $20 \mathrm{mg} / 1$ Peroxidase katalytisch vollständig umgesetzt. Die Absorbanz der Testlösung wird bei der Wellenlänge $370 \mathrm{~nm}$ gemessen. An Hand einer mitgeführten Standardreihe kann die $\mathrm{H}_{2} \mathrm{O}_{2}$ Konzentration in der Probe direkt abgelesen werden. Die Nachweisgrenze dieses enzymatischen $\mathrm{H}_{2} \mathrm{O}_{2}$-Tests beträgt bei einem Meßbereich von $0-40 \mu \mathrm{mol} / \mathrm{l}$ etwa $2 \mu \mathrm{mol} / 1 \mathrm{H}_{2} \mathrm{O}_{2}$.

\section{Peroxidaseaktivität in Abhängigkeit vom Puffersystem}

Unter sonst identischen Testbedingungen $(3,0 \mathrm{mmol} / 1$ $\mathrm{H}_{2} \mathrm{O}_{2}, 1,0 \mathrm{mmol} / \mathrm{l} 3,3^{\prime}, 5,5^{\prime}$-Tetramethylbenzidin $\mathrm{pH}$ 3,95 und $25^{\circ} \mathrm{C}$ ) wird mit je $0,2 \mathrm{~mol} / 1$ Kaliumcitrat und Kaliumacetat eine gleich hohe Peroxidaseaktivität gemessen, während die Peroxidase in $0,2 \mathrm{~mol} / \mathrm{l}$ Kaliumphosphat um 10-15\% weniger aktiv ist.

Der Einfluß der Kaliumcitratkonzentration auf die Peroxidaseaktivität wurde untersucht und dabei festgestellt, daß mit steigendem Gehalt an Kaliumcitràt in der Testlösung sowohl die Aktivität wie auch die Inaktivierungsgeschwindigkeit der Peroxidase zunehmen. Bei einer 30-minütigen Reaktionsdauer wird die höchste Peroxidaseaktivität mit einer Kaliumcitratkonzentration zwischen $100-200 \mathrm{mmol} / \mathrm{l}$ erreicht.

\section{Peroxidaseaktivität in Abhängigkeit der} Reaktionstemperatur

Die katalytische Aktivität der Enzyme ist allgemein sehr stark von der Reaktionstemperatur abhängig. So bewirkt generell eine Erhöhung der Temperatur um $1{ }^{\circ} \mathrm{C}$ innerhalb des Temperaturbereichs von $0^{\circ} \mathrm{C}$ bis etwa $45^{\circ} \mathrm{C}$ einen Anstiẹg dèr enżymatischen Reaktionsgeschwindigkeit um 5-15\%. Daher ist für die Enzymbestimmung die genaue Einhaltung der vorgeschriebenen Inkubationstemperatur eine wesentliche Voraussetzung für korrekte Resultate.

Die katalytische Reaktion der Peroxidase mit den Substraten $\mathrm{H}_{2} \mathrm{O}_{2}$ und 3,3',5,5'-Tetramethylbenzidin weist eine völlig anders geartete Temperaturabhängigkeit auf, wie dies aus den Resultaten der Abbildung 10 deutlich wird. Unter den gegebenen Reaktionsbedingungen wird die höchste Peroxidaseaktivität bei einer Inkubationsdauer von 30 Minuten im Temperaturbereich von $20-25^{\circ} \mathrm{C}$ gemessen. Generell ist festzustellen, dả $ß$ mit steigender Reaktionstemperatur die Zeit-Aktivitätskurven der Peroxidase im Laufe der 30-minütigen Reaktionsdauer immer stärker abflachen. Diese komplexe Temperaturabhängigkeit der

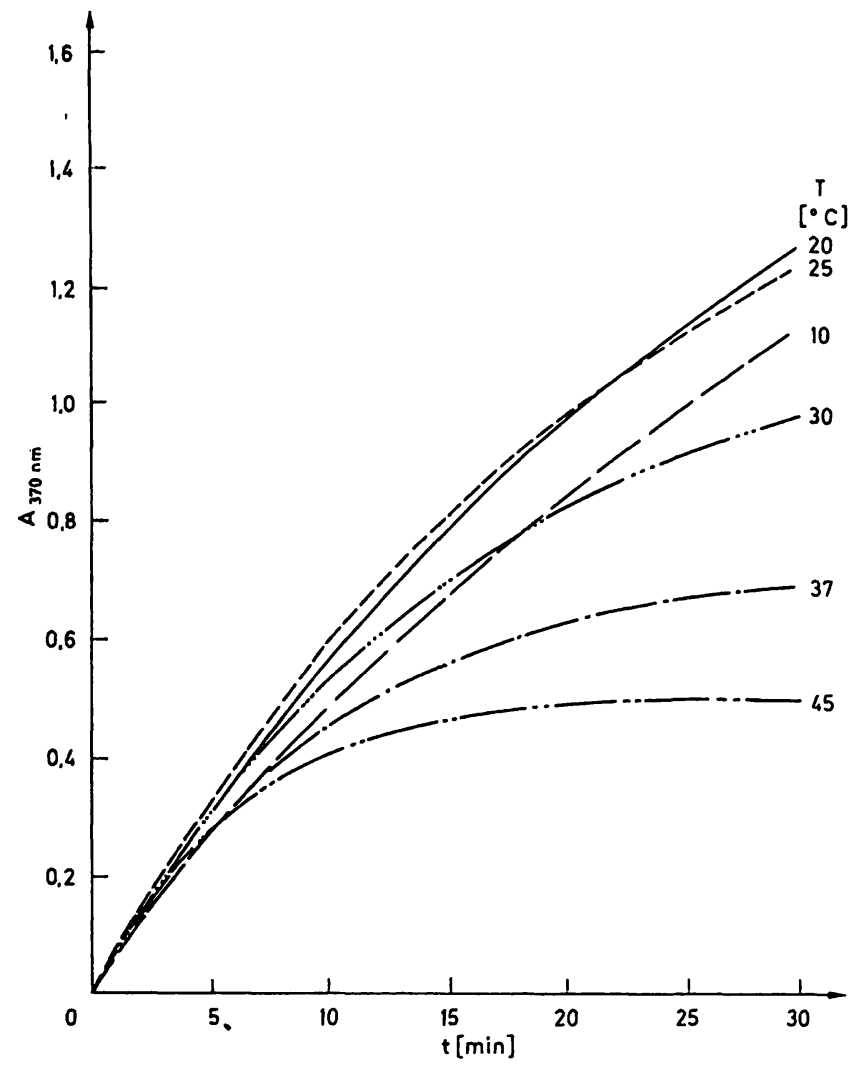

Abb. 10. Peroxidaseaktivität in Abhängigkeit zur Inkubationstemperatur.

$150 \mathrm{ng} / \mathrm{l}$ Peroxidase wurde bei unterschiedlichen Reaktionstemperaturen in $0,2 \mathrm{~mol} / \mathrm{l} \mathrm{Kaliumcitratpuffer}$ vom $\mathrm{pH} 3,95$ mit $3 \mathrm{mmol} / \mathrm{l} \mathrm{H}_{2} \mathrm{O}_{2}$ und $1,0 \mathrm{mmol} / \mathrm{l}$ $3,3^{\prime}, 5,5^{\prime}$-Tetramethylbenzidin inkubiert, wobei während der 30-minütigen Reaktionsdauer die Absorbanz bei der Wellenlänge $370 \mathrm{~nm}$ laufend gemessen wurde.

Peroxidaseaktivität wird dominiert von der Enzyminaktivierung, die mit steigender Temperatur wesentlich schneller zunimmt als die katalytische Reaktion des noch aktiven Enzyms.

Auf Grund der aufgezeigten Temperaturabhängigkeit kann die Peroxidaseaktivität unter den gegebenen Reaktionsbedingungen im Temperaturbereich von $20-25^{\circ} \mathrm{C}$ durchgeführt werden.

Reagens zum Abstoppen der Peroxidaseaktivitä t

Die Peroxidase wird häufig als Markierungsenzym bei enzym-immunologischen Tests verwendet. Bei der Durchführung dieser Tests erfolgt die Bestimmung der Peroxidase nicht nach der kontinuierlichen, sondern nach der Zwei-Punkt-Methode. Bei diesem Vorgehen ist es vorteilhaft, wenn nach einer bestimmten Reaktionsdauer die peroxidatische Aktivität gestoppt und die während der Indikatorreaktion entstandene Farbintensität soweit stabilisiert werden kann, so daß die photometrische Messung - innerhalb einer gewissen Dauer - zeitunabhängig durchgeführt werden kann. 
Durch Senken des pH-Wertes der Testlösung auf pH 1,0 wird die katalytische Aktivität der Peroxidase sogleich und vollständig gestoppt und das gebildete, blaugefärbte Radikalkation (II) wird zum gelbgefärbten $3,3^{\prime}, 5,5^{\prime}$-Tetramethyl-1,1'-diphenochinon-4,4'-diimoniumion (III) übergeführt, das eine wesentlich höhere Absorption aufweist und bei der gebräuchlichen Wellenlänge $450 \mathrm{~nm}$ photometrisch gemessen werden kann. Eine ausreichende pH-Senkung und damit zusammenhängend die größtmögliche Steigerung der Farbintensität (vgl. Abb. 5) kann nur mit starken Säuren erreicht werden. Von den geprüften Abstoppreagenzien $\left(\mathrm{HCl}, \mathrm{H}_{2} \mathrm{O}_{2}, \mathrm{HNO}_{3}, \mathrm{H}_{3} \mathrm{PO}_{4}\right)$ wird mit Schwefelsäure die beste Farbstabilität erreicht. Die zum Abstoppen der Peroxidaseaktivität verwendeten Säuren müssen frei sein von Eisenionen. Die zum Erreichen des pH-Wertes von 1,0 erforderliche Schwefelsäurekonzentration ist abhängig vom Volumenverhältnis der Testlösung zum Abstoppreagens.

Die katalytische Aktivität der Peroxidase kann auch durch Erhöhung der $\mathrm{H}_{2} \mathrm{O}_{2}$-Konzentration in der Testlösung abgestoppt werden. Bei Anwesenheit von $100 \mathrm{mmol} / 1 \mathrm{H}_{2} \mathrm{O}_{2}$ wird die Peroxidase praktisch sogleich und vollständig inaktiviert, die Farbintensität des Radikalkation (II) wird dadurch stabilisiert und die Absorbanz kann innerhalb von 60 Minuten bei den Wellenlängen $370 \mathrm{~nm}$ oder $650 \mathrm{~nm}$ photometrisch gemessen werden.

Optimierte Bestimmungsmethode der Peroxidase

Auf Grund der experimentellen Daten wird folgende Testmethode zur quantitativen Bestimmung der Peroxidase vorgeschlagen: Die zu bestimmende Peroxidase $(0-200 \mathrm{pg})$ wird in $1,0 \mathrm{ml}$ Substratpuffer $(0,2 \mathrm{~mol} / \mathrm{l}$ Kaliumcitrat vom pH $3,95 \mathrm{mit} 3 \mathrm{mmol} / 1 \mathrm{H}_{2} \mathrm{O}_{2}$ und 1,0 $\mathrm{mmol} / 13,3^{\prime}, 5,5^{\prime}$-Tetramethylbenzidin) während $30 \mathrm{Mi}$ nuten bei $20-25^{\circ} \mathrm{C}$ lichtgeschützt inkubiert. Nach Ablauf der Reaktionszeit werden zum Abstoppen der peroxidatischen Aktivität $1,0 \mathrm{ml} 1 \mathrm{~mol} / \mathrm{l}$ Schwefelsäure zugemischt und die Farbintensität innerhalb von 60 Minuten bei der Wellenlänge $450 \mathrm{~nm}$ photometrisch gemessen.

\section{Literatur}

1. Holland, V., Saunders, B., Rose, F. \& Walpole, A. (1974) Tetrahedron 30, 3299-3302.

2. Garner, R., Walpole, A. \& Rose, F. (1975) Cancer Letters $1,39-42$.

3. Standefer, J. \& Vanderjagt; D. (1977) Clin. Chem. 23, 749-751.

4. Lijana, R. \& Williams, M. (1979) J. Lab. Clin. Med. 94, $266-276$.
Abbildung 11 zeigt die lineare Proportionalität der im Test eingesetzten Peroxidasemenge zur gemessenen Farbintensität nach der 30-minütigen Enzymreaktion, wobei einerseits vor der Zugabe von Schwefelsäure die Absorbanz bei der Wellenlänge $370 \mathrm{~nm}$ und andererseits nach dem Abstoppen der Peroxidaseaktivität die Farbintensität bei der' 'Wellenlänge $450 \mathrm{~nm}$ photometrisch gemessen wurde. Bei einem Meßbereich von $0-200 \mathrm{pg}$ Peroxidase kann unter den gegebenen Testbedingungen das Enzym mit einer Nachweisgrenze von etwa $10 \mathrm{pg}$ quantitativ bestimmt werden. Die Reproduzierbarkeit innerhalb einer Meßserie kann mit einem Variationskoeffizient von $0,7-4,5 \%$ als gut bezeichnet werden.

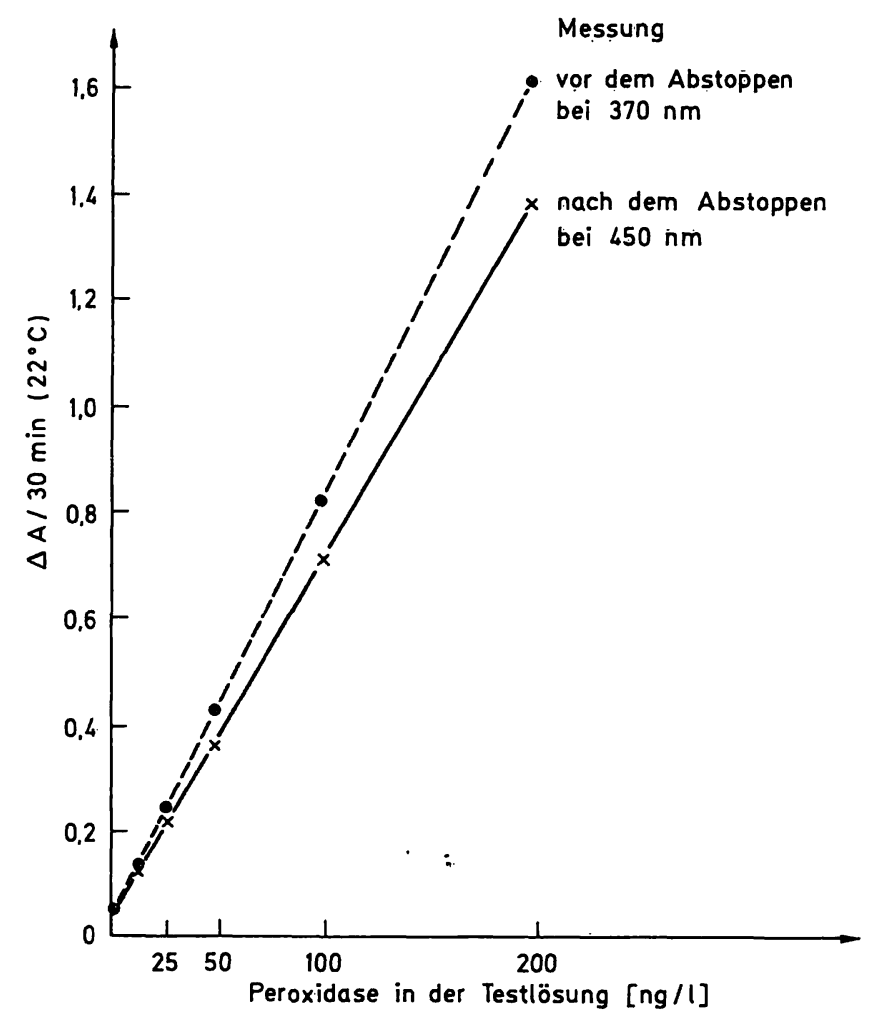

Abb. 11. Abhängigkeit der Absorptionsdifferenz von der eingesetzten Peroxidasekonzentration und der Testmethode.

Die angegebene Peroxidasemenge wurde in $0,2 \mathrm{~mol} / \mathrm{l}$ Kaliumcitratpuffer vom pH 3,95 mit $3 \mathrm{mmol} / 1 \mathrm{H}_{2} \mathrm{O}_{2}$ und $1,0 \mathrm{mmol} / 13,3^{\prime}, 5,5^{\prime}$-Tetramethylbenzidin während 30 Minuten bei $22^{\circ} \mathrm{C}$ inkubiert. Nach der Messung der Absorbanz bei der Wellenlänge $370 \mathrm{~nm}(---)$ wurde die katalytische Reaktion durch Zugabe von $1,0 \mathrm{ml} 0,5 \mathrm{~mol} / 1$ Schwefelsäure pro $1,0 \mathrm{ml} \cdot$ Testlösung abgestoppt und die Farbintensität nochmals bei der Wellenlänge $450 \mathrm{~nm}$ gemessen. (

5. Bos, E., van der Doelen, A., van Rooy, N. \& Schuurs, A. (1981) J. Immunoassay 2, 187-204.

6. Josephy, P., Eling, Th. \& Mason, R. (1982) J. Biol. Chem. $257,3669-3675$.

Dr. H. Gallati

Zentrale Forschungseinheiten

F. Hoffmann-La Roche \& Co. AG

CH-4000 Basel if 